\title{
Augmentation of transfers for a quadriplegic patient using an implanted FNS system. Case report
}

\author{
E B Marsolais MD, PhD ${ }^{1}$ A Scheiner PhD PE ${ }^{2} \mathrm{P}$ C Miller MEd, ${ }^{3}$ R Kobetic MS,${ }^{4}$ \\ J Jacobs Daly $\mathrm{MS}^{5}$ \\ ${ }^{1}$ Chief, Rehabilitation Medicine Service and Motion Study Laboratory, VA Medical \\ Center, 10701 East Boulevard, Cleveland, Ohio 44106, USA and Associate Professor of \\ Orthopaedics and Director of the Division of Rehabilitation, Department of Orthopaedics, \\ Case Western Reserve University School of Medicine, Cleveland, OH, USA; ${ }^{2}$ Research \\ Engineer, ${ }^{3}$ Exercise Physiologist, ${ }^{4}$ Supervisory Biomedical Engineer, ${ }^{5}$ Coordinator, \\ Clinical Research, VA Medical Center, Cleveland, Ohio, USA.
}

\begin{abstract}
A 22 year old man with incomplete quadriplegia (C6-7) was unable to perform either a sliding or a pivot transfer. He was instrumented with an implanted functional neuromuscular stimulation (FNS) system, radio frequency-linked to a belt-worn controller. The system activated eight muscles selected from among quadriceps, hamstrings, posterior portion of the adductor magnus, gluteus maximus, and erector spinae, bilaterally. The two-stage implantation procedure included electrode implantation with percutaneous leads followed by stimulator implantation and removal of the percutaneous leads. All implants were well tolerated with no adverse effects. The subject was able independently to put on the external controller portion of the system and to perform a standing pivot transfer with only standby assistance. An unexpected outcome of the FNS system use was increased voluntary upper body strength that resulted in improvement of the sliding transfer from 'inability' to 'independent'.
\end{abstract}

Keywords: quadriplegia; functional neuromuscular stimulation; FNS; neuroprosthesis; functional restoration.

\section{Introduction}

People with impaired arm and hand function in addition to lower extremity paralysis often face severe mobility problems including inability to perform a sliding transfer. ${ }^{1}$ They are confined to a bed or chair until help in moving can be provided by a sufficiently strong attendant or a mechanical lift. Some individuals require institutionalization because family carers are not able to provide the level of assistance needed for the transfer. Professional carers are particularly careful about performing repeated maximum effort transfers for quadriplegic persons. ${ }^{2}$ In some institutions, professional carers limit the number of bed-to-chair transfers provided for a quadriplegic patient because of the difficulty of the transfer. To the detriment of his general health, the patient may opt to stay in the wheelchair in the same position all day rather than face being bedridden for much of the day. Inactivity leads to physical problems including skin deterioration, pressure sores, and a negative emotional impact. Care of this disabled group is costly for both the individuals and society.

The widely available systems for transfer for the quadriplegic person are lifts. Other potential systems are braces and, both percutaneous and surface electrode, functional neuromuscular stimulation (FNS) systems. None of these systems is widely used, each for its own reasons. Lifts are large and cumbersome and not conveniently available in all settings. Braces require that assistance be provided first to apply the brace and then to stand the quadriplegic person before the hip and knee can be locked. ${ }^{3}$ This is so difficult that such braces are used only in 
partially paralyzed or incomplete quadriplegic patients. The major disadvantages of surface electrode FNS systems for quadriplegic individuals are the impossibility of personally putting on the electrodes, and the variable and sometimes inadequate muscle contractions. ${ }^{4,5}$ In our laboratory we have tested a FNS system using percutaneous intramuscular (IM) electrodes and an external stimulator. Disadvantages of this FNS system are the need to take care of the lead exit sites, the possibility of infection at lead exit sites, and mechanical problems with wires connecting the skin sites to the stimulator. ${ }^{6,7}$

We have investigated a system that was developed at the Cleveland VA Medical Center and Case Western Reserve University (VA-CWRU) in which both the stimulator and electrodes are implanted. Controlled by a radio frequency link, this FNS system does not have most of the disadvantages of lifts, braces, surface FNS systems, or of the FNS system with percutaneous leads. However, the radio frequency-linked system poses risks associated with the implantation surgery such as infection ${ }^{8}$ and the possibility of system failure (Keith MW, personal communication). This paper reports a case study of the first implementation for pivot transfer of the VA-CWRU neuroprosthetic standing system in a quadriplegic patient.

\section{Materials and methods}

The subject was a 22 year old quadriplegic man (Frankel C6-7), 19 months post injury, with partial sensation, who could be brought to a standing position with the maximal assistance of one helper. He had manual muscle test grades of: trunk and left hip 3/5; right leg $0 / 5$; left knee and ankle $2 / 5$; shoulder, elbow and wrist $4 / 5$; and fingers $2 / 5$. He was unable to perform a sliding or a pivot transfer despite many months of therapy. He observed paraplegic patients becoming able to stand and walk with electrical stimulation and requested to be evaluated as a potential subject using electrical stimulation to assist him with a standing transfer.

Implementation of the FNS system was done in two stages. First, the quadriceps, hamstrings, posterior adductor magnus, gluteus maximus and erector spinae muscles were implanted with IM electrodes connected to percutaneous leads (Fig 1). This stage of the procedure enabled testing of the implanted electrodes and exercise of the instrumented muscles prior to implantation of the radio frequency-linked stimulator. It also allowed electrode stabilization; our earlier work ${ }^{9}$ showed that many electrode failures occurred in the first 3 months after implantation.

Electrodes were implanted as follows. First, a 26-gauge stimulating probe was used to locate the desired site of stimulation. Then, a larger sheath was passed over the probe, allowing precise substitution of the probe with an intramuscular electrode whose lead was enclosed in Silastic ${ }^{\mathrm{TM}}$ tubing (Dow Corning Corp, Midland, MI, USA) (closed helix electrode) and terminated with a connector. The electrode lead with an attached connector was then passed subcutaneously to the abdomen where it was attached to a temporary percutaneous lead; each connection was made through a $1 \mathrm{~cm}$ incision which was closed with a single stitch that was removed after 1 week. The percutaneous lead was then passed subcutaneously to the groin and brought through the skin. This lead was attached to a miniature connector for easy hook-up to an external stimulator during exercise and transfer training. The first stage of implantation required a series of 17 outpatient sessions of approximately 3.5 hours each, including all preparation and cleaning time.

Using the external stimulator, the subject performed FNS exercise for 1 hour per day and stood with FNS assistance 3 days per week for many repetitions of up to 3 minutes each totaling about 15 minutes per session. After 36 weeks, when testing showed sufficient function, the best set of eight electrodes was chosen for the radio frequency-linked FNS system by the following method. The quadriceps and the erector spinae muscles formed the basis of the set of electrodes. Two of the three remaining muscles, bilaterally, were added to the basic set and the standing pivot transfer was evaluated. This was repeated for all three 
(INTER-CONNECTOR)

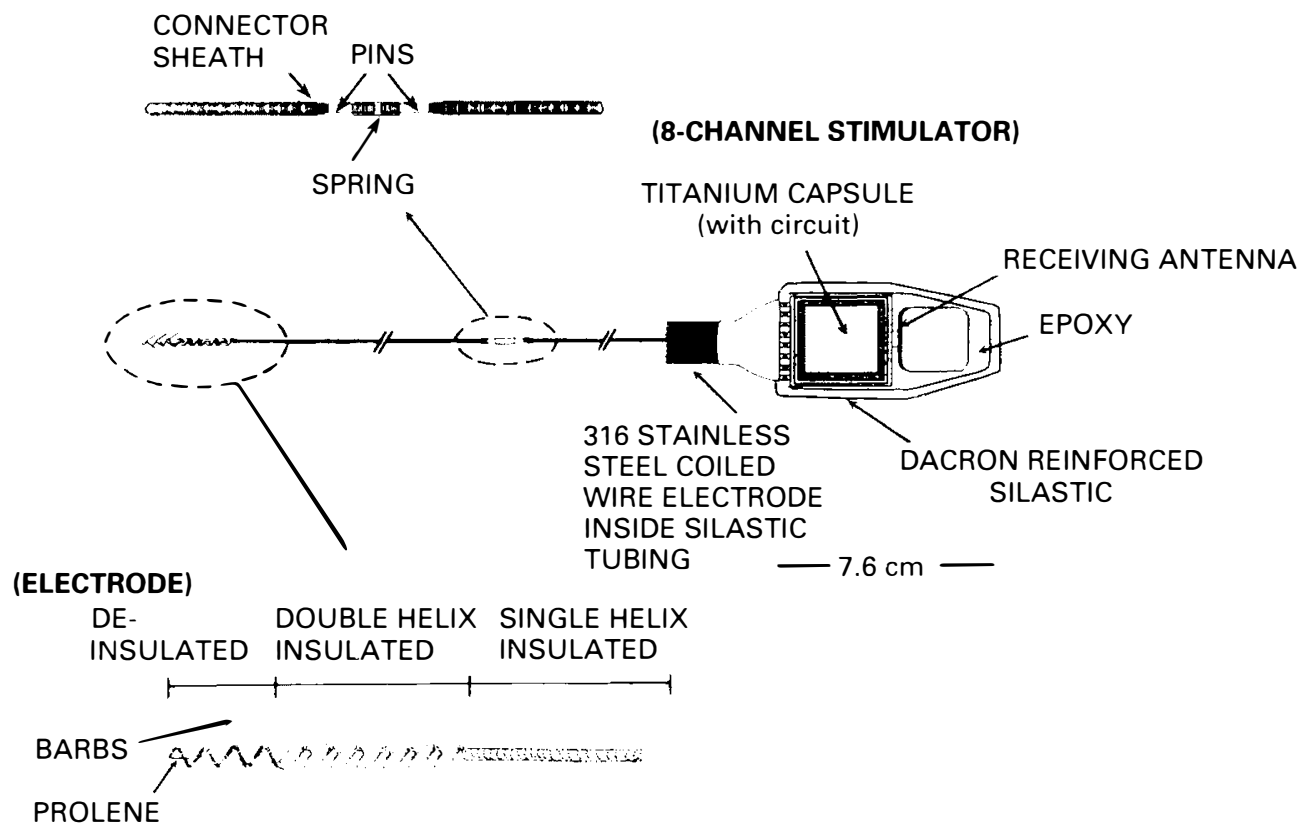

Figure 1 Diagram of implanted portions of the radio frequency-linked FNS lower extremity neuroprosthetic system.

combinations and the one with the best functional transfer outcome as judged by the subject and the research team was selected.

The temporary percutaneous components of the chosen electrodes and all components of any rejected electrodes were removed 2 weeks before the second stage of implantation to allow time for healing. Surgery was performed under aseptic conditions as in total joint surgery ${ }^{10}$ and using local anesthesia. Any residual portions of the percutaneous leads were removed from the abdomen through separate $1 \mathrm{~cm}$ incisions, leaving the eight chosen electrodes and closed helix leads. Location of electrode lead connectors was facilitated with a few seconds of fluoroscopy. Through a $3.5 \mathrm{~cm}$ incision, the stimulator was placed subcutaneously in the lower left abdomen and was connected to the closed helix leads.

The stimulator was the same 8-channel, microprocessor-controlled device (Fig 1) previously used for control of grasp and release functions of the hand in quadriplegic patients. ${ }^{11} \mathrm{~A}$ hernia belt was modified and used to keep the external transmitting coil in the proper position in relation to the receiving coil of the implanted stimulator (Fig 2). The patient activated the stimulator with a walker mounted hand switch.

Evaluation of the FNS system was based on five outcome measures: (1) ease of implantation, (2) physical and mental responses of the patient to the procedure, (3) muscle response produced by the implanted system, (4) functional capability of the patient, and (5) system performance. Ease of implantation was evaluated by assessment by the surgeon, the orthopedic nurse, and biomedical engineering staff. The subject's physical responses to the procedure were evaluated by the same staff members, who monitored standard perioperative and intraoperative factors: $\mathrm{BP}$, pulse, and patient communication. Mental responses were evaluated by the treatment team using personal interaction. Muscle response was tested with manual muscle testing and with the Cybex II $^{\mathrm{TM}}$ dynamometer (Lumex, Ronkonkoma, NY, USA). Functional 
a

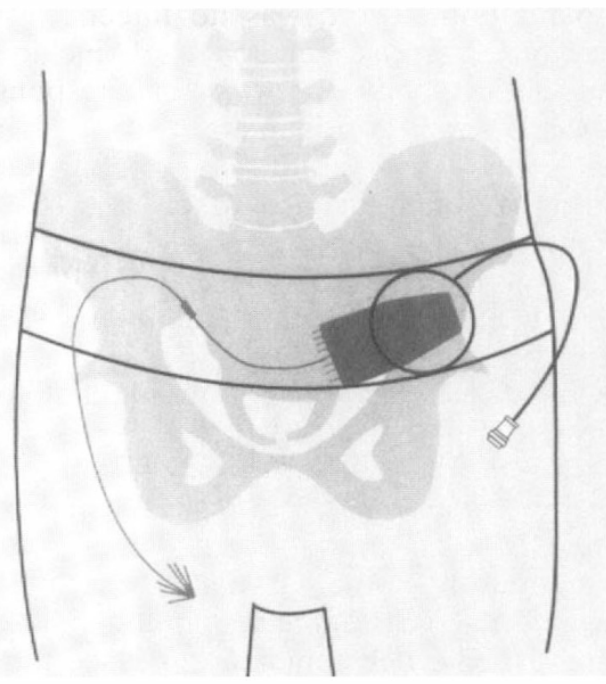

b

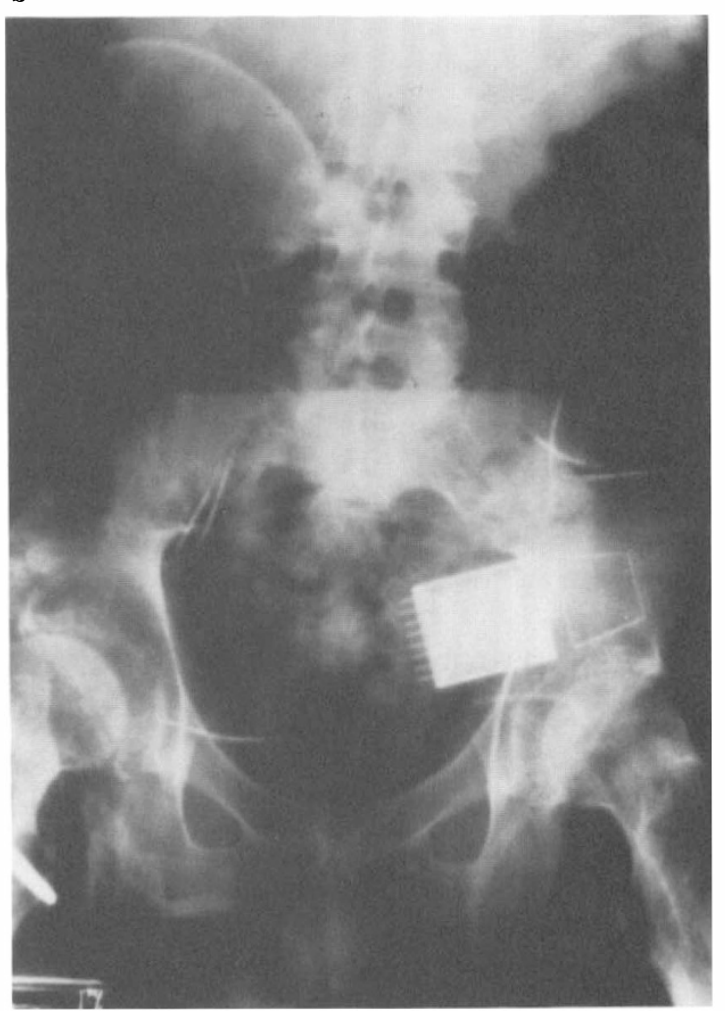

Figure 2 (a) Diagrammatic presentation of hernia belt in place on subject. (b) X-ray film of pelvis showing 8-channel stimulator.

capability and safety were assessed by a physical therapist, a biomedical engineer, an exercise physiologist and a medical rehabilitation specialist using video cameras; and by structured interview of the subject. Upper body support was measured with a force plate (AMTI, Newton, MA, USA) and an instrumented walker (Skoch MJ, unpublished project). System performance in the laboratory were evaluated by direct observation and video documentation; system performance and practicality outside the laboratory were evaluated by a structured interview of the subject and by evaluation by a physical therapist at home.

\section{Results}

The patient was able to stand independently after 6 weeks (Fig 4) and to do an independ- ent standing pivot transfer with standby assist at 12 weeks. No change in muscle torques as measured using the percutaneous system with the dynamometer was noted on the day after implantation of the stimulator and at monthly follow up testing. The subject stood for up to 3 minutes and supported at least $60 \%$ of his body weight on his legs. His resting heart rate of $56 \mathrm{bpm}$ increased to $86 \mathrm{bpm}$ during the 30 second transfer and returned to the resting rate by 4 minutes after completion. After 1 year of electrical exercise, evaluation of isometric fatigue of the hip extensors showed $50 \%$ of the maximal force after 100 seconds of continuous stimulation; testing of the quadriceps showed over $50 \%$ of the peak force remaining after 60 minutes of cyclic stimulation (1 s on, $3 \mathrm{~s}$ off). The graph in Fig 3 illustrates the maximum muscle torques taken at an angular velocity of 60 degrees/ 


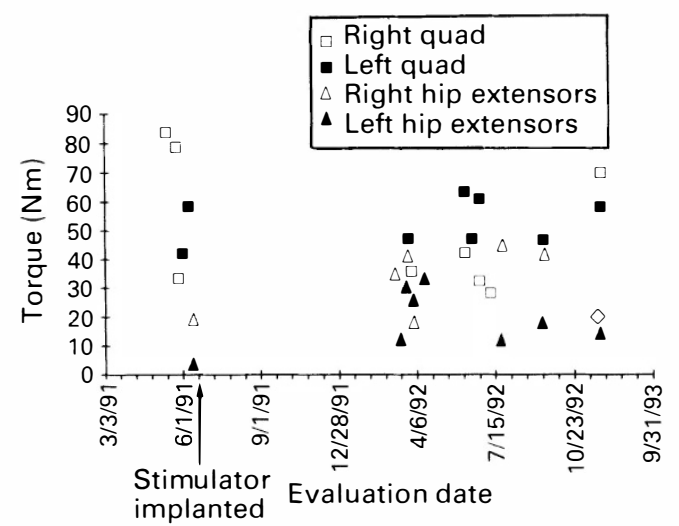

Figure 3 Cumulative knee and hip extension torque data.

second for the quadriceps and isometrically at 45 degrees of hip flexion for the hip extensors.

Although the subject had some intact sensation, he noted no significant discom-

a

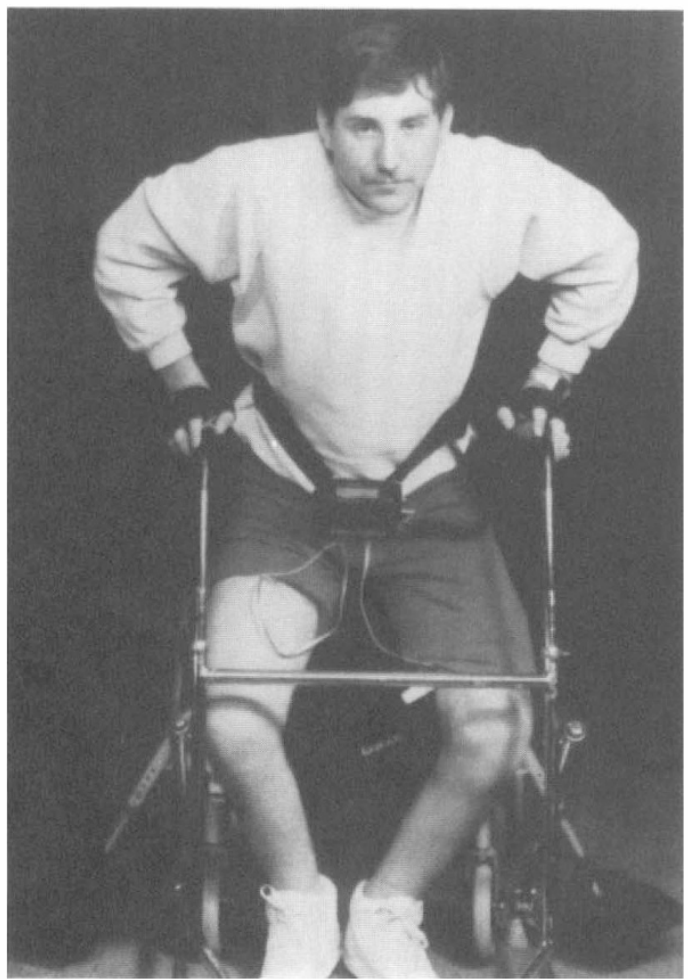

fort during either phase of the system implantation. There was no infection and very little response of any kind, including pain, skin response, or deep tissue response, to the procedures. The stimulator was implanted under local anesthesia at 36 weeks during a 3.5 hour procedure selected as inpatient for the subject's convenience. But, based on its medical requirements, it could have been an outpatient procedure. The subject was able to use the system for exercise on the day following implantation of the stimulator.

At 32 weeks from implantation of the first electrode the subject fulfilled the criteria for home use of the system for transfers. After implantation of the stimulator, putting on the external parts of the system took about 4 minutes. The belt remained in place for as long as a full day without needing adjustment. Some initial radio frequency coupling problems due to low battery voltage were found and solved. The walker mounted

b

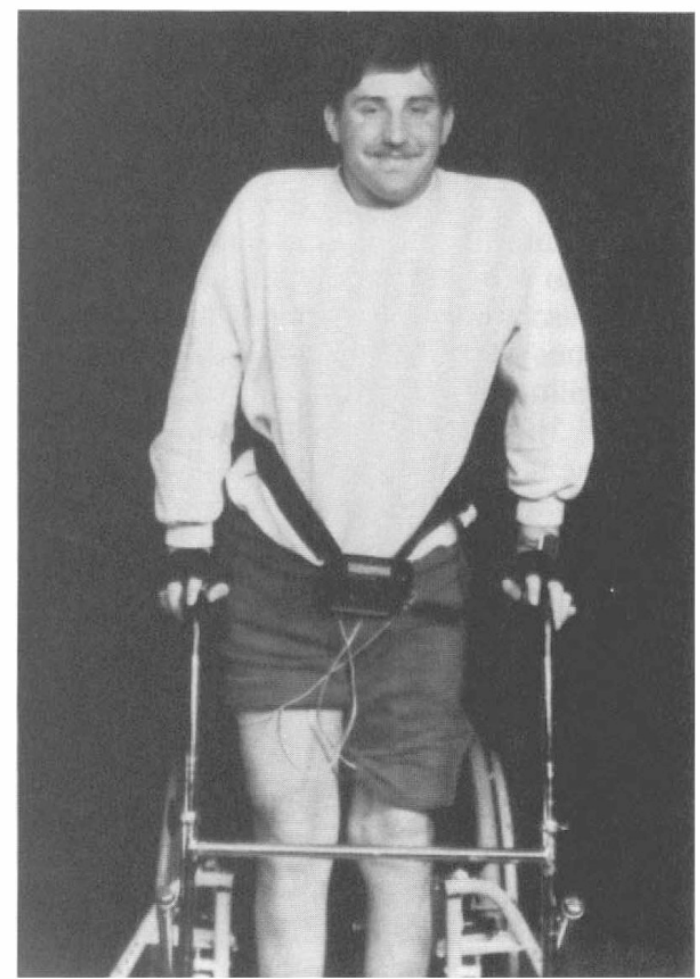

Figure 4 Quadriplegic subject using FNS system for standing. 
hand switch proved inadequate for uses other than the transfer. The switch was replaced with a control switch worn on the hand and utilizing residual motion in the left fifth digit. Both the subject and the research team agreed that the FNS system was of major value for exercise and transfers. The system was demonstrated to be practical and safe for transfers at home.

\section{Discussion}

The initial response of this patient to permanent FNS implantation was positive. First, he accomplished the pivot transfer easily and safely with the 8-channel FNS assist. Second, the simple two stage implantation technique was well tolerated. Seven of the eight electrodes were well established prior to insertion of the stimulator. The weak posterior adductor electrode was accepted prior to implantation since it did not impair his transfer ability. Preimplantation of the electrodes obviated the need for lengthy surgery with the attendant patient discomfort and anesthetic and infection risks. ${ }^{12}$ Certainly there was some risk from the percutaneous interface but we have documented this to be small $^{8,9}$ and the percutaneous leads were removed prior to the implantation of the stimulator. The time needed to implant the percutaneous system is unreasonable for most patients. New techniques are being developed which should allow the electrodes to be implanted in two 3.5 hour outpatient visits. ${ }^{13}$ The components of the implanted system have demonstrated initial stability: six electrodes functioned adequately with an average time since implantation of 106 weeks; one posterior adductor electrode was inadequate at the stimulator implantation and the remaining posterior adductor electrode was no longer producing the desired response 44 weeks after implantation. The failure did not adversely affect the pivot transfer ability. The stimulator has functioned adequately for 58 weeks. The abdominal site has been well tolerated both physiologically and cosmetically.

The FNS system was found practical and safe for home use. The system enabled the patient to strengthen his residual voluntary muscles, both stimulated and nonstimulated. As stated above, he did not increase the strength of the stimulated muscle but increased fatigue resistance allowing longer standing. Through increase in voluntary strength he became able to do an unassisted sliding transfer which was faster and simpler than a pivot transfer. He therefore uses the unassisted sliding transfer whenever possible. He continues to use the stimulator for exercise to maintain leg strength and for selected activities at home such as difficult sliding transfers. A similar result has been reported after use of a surface electrode FNS system for gait in patients with incomplete spinal cord injury ${ }^{14}$ and reflects the therapeutic aspect of FNS. The question of attempting this study with surface electrodes might be considered, but this patient could not apply surface electrodes independently. In addition, our experience with paraplegic individuals possessing normal hands indicates that daily application of surface electrodes is extremely difficult.

The therapeutic effect of the stimulation through increase of muscle bulk and strength, increase of bone mineral density, and increase of aerobic capacity has been previously described in our publications concerning paraplegia. ${ }^{6}$ It certainly was possible to predict this therapeutic effect on the patient prior to the implantation procedure, but the extent to which he would be able to integrate this effect into his personal mobility capability could not be determined prior to the implantation. The implantation gave him a simple way to do large amounts of electrical exercise of specific muscles which is currently not readily available in any other form.

\section{Conclusions}

We presented the case history of a quadriplegic individual with an incomplete lesion who was incapable of a sliding or pivot transfer who became capable of a standing pivot transfer after implantation and conditioning of eight muscles activated with FNS. Implantation of both the percutaneous and permanent versions of the FNS system was 
well tolerated. We also demonstrated that while this system was practical and safe for functional home use, gains in voluntary muscular strength associated with the electrical exercise resulted in reduced need for direct functional use of the system.

\section{Acknowledgements}

The authors thank Jane Marek RN, MSN for operative assistance, Anita Maximin PsyD for psychological assessment, Carole Kantor MS for editing, and Rockney Shepheard for creating the figures.

\section{References}

1 Guttmann L (1973) Spinal Cord Injuries. Comprehensive Management and Research. Blackwell Scientific Publications, Oxford.

2 Sorrentino S (1987) Mosby's Textbook for Nursing Assistants. Mosby Co, St Louis, MO.

3 Kantor C, Andrews BJ, Marsolais EB et al (1993) Report on a conference on motor prostheses for workplace mobility of paraplegic patients in North America. Paraplegia 31: 439-456.

4 Chaplin E, Habasevich R (1992) Ambulation with functional electrical stimulation (FES) in individuals with paraplegia and incomplete quadriplegia. Neurology 42 (Suppl 3): 638.

5 Petrofsky JS, Phillips CA, Douglas R, Larson P (1986) A computer controlled walking system: The combination of an orthosis with functional electrical stimulation. J Clin Eng 11: 121-133.

6 Marsolais EB, Kobetic R, Chizeck HJ, Jacobs JL (1991) Orthoses and electrical stimulation for walking in complete paraplegia. J Neurol Rehabil 5: 13-22.

7 Keith MW, Peckham PH, Thrope GB et al (1988) Functional neuromuscular stimulation neuroprostheses for the tetraplegic hand. Clin Orthop 233: 25-33.

8 Marsolais EB, Kobetic R (1986) Implantation techniques and experience with percutaneous intramuscular electrodes in the lower extremities. J Rehabil Res Dev 23: 1-8.

9 Scheiner, Polando G, Marsolais EB (1994) Design and clinical application of a double helix electrode for functional electrical stimulation. IEEE Trans Biomed Eng $4 \mathbf{1}$.

10 Salvati EA, Robinson RP, Zeno SM et al (1982) Infection rates after 3175 total hip and total knee replacements performed with and without a horizontal unidirectional filtered airflow system. J Bone Joint Surg Am 64: 525-535.

11 Smith B, Peckham PH, Keith MW, Roscoe DD (1977) an externally powered, multichannel, implantable stimulator for versatile control of paralyzed muscle. IEEE Trans Biomed Eng 34: 499-508.

12 Rushton DH, Brindley GS, Polkey CK, Browning GV (1989) Implant infections and antibiotic impregnated silicone rubber coating. J Neurol Neurosurg Psychiatry 52: 223-229.

13 Osman SG, Marsolais EB (1994) Endoscopic electrode implantation for enhanced stimulation of the hamstring branches of the sciatic nerve. Arthroscopy 10.

14 Granat MH, Ferguson ACB, Andrews BJ, Delargy M (1993) The role of functional electrical stimulation in the rehabilitation of patients with incomplete spinal cord injury observed benefits during gait studies. Paraplegia 31: 207-215. 